\title{
Relevância Diagnóstica da Massagem do Seio Carotídeo Durante Teste de Inclinação
}

\author{
Diagnostic Relevance of the Carotid Sinus Massage During a Head Up Tilt Table Test (HUTT) \\ Marcos Benchimol1,2, Ricardo Oliveira-Souza

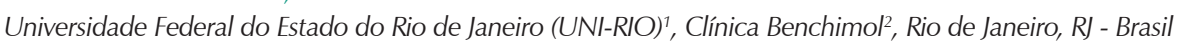

\section{Resumo}

Fundamento: As síncopes por hipersensibilidade do seio carotídeo (HSC) podem ser pouco diagnosticadas por causa da baixa desconfiança diagnóstica ou do medo de complicações da própria massagem do seio carotídeo.

Objetivo: Investigar o papel da hipersensibilidade do seio carotídeo (HSC) em desmaios não-convulsivos e quedas inexplicadas.

Métodos: Duzentos e cinqüenta e nove pacientes com idade média de $50 \pm 24$ anos, encaminhados para investigação de desmaios não-convulsivos e quedas inexplicadas, foram submetidos ao Teste da Mesa Inclinada (TI) sem ("passivo") e com provocação farmacológica ("sensibilizado"). Exames clínicos e complementares não revelaram causa óbvia para as queixas. Cinqüenta e cinco voluntários com idade média de $57 \pm 21$ anos sem história de epilepsia, desmaios e quedas serviram de controles. Todos os participantes foram submetidos ao TI precedido por estimulação digital dos seios carotídeos a zero e a $60^{\circ}$.

Resultados: A estimulação dos seios carotídeos foi positiva em 4 controles. O diagnóstico clínico de HSC foi endossado por resposta positiva à estimulação carotídea a $60^{\circ} \mathrm{em} 28$ pacientes, em sua maioria homens idosos. A estimulação carotídea foi positiva a $0^{\circ}$ em apenas três desses pacientes. Não houve diferença estatística no TI entre os pacientes com e sem HSC.

Conclusão: A estimulação dos seios carotídeos a $60^{\circ}$ deve ser incluída na avaliação rotineira de pacientes com desmaios não-convulsivos ou quedas inexplicadas, uma vez que os exames cardiovasculares de rotina, incluindo o TI, não foram úteis para estabelecer o diagnóstico nesses casos. Novos estudos em indivíduos normais são necessários para estabelecer o significado da resposta positiva à estimulação carotídea na ausência de história de desmaios ou quedas. (Arq Bras Cardiol 2008; 90(4): 288-292)

Palavras-chave: Síncope, hipersensibilidade, seio carotídeo, teste da mesa inclinada.

\section{Summary}

Background: Syncope due to carotid sinus hypersensitivity (CSH) may be underdiagnosed due to a low level of diagnostic suspicion and fear of complications caused by massage of the carotid sinus.

Objective: To investigate the relevance of carotid sinus massage in the diagnosis of non-convulsive faints and unexplained falls.

Methods: Two-hundred and fifty-nine patients with a mean age of $50 \pm 24$ years referred for the investigation of non-convulsive faints or unexplained falls were evaluated with a head up tilt table test (HUTT) without ("passive") and with pharmacological stimulation. Clinical and laboratory work-up did not reveal an obvious cause for the complaints. Fifty-five volunteers with a mean age of $57 \pm 21$ years with no history of seizures, faints or falls were used as controls. All participants underwent a HUTT preceded by digital stimulation of each carotid sinus both at zero and $60^{\circ}$

Results: Carotid sinus massage was positive in 4 controls. The relevance of CSH in the evaluation of syncope was supported by a positive response to carotid sinus massage at $60^{\circ}$ in 28 patients, most of whom were elderly men. Carotid sinus massage was positive at $0^{\circ}$ in only three of such patients. The results of the HUTT did not show statistical difference between patients with and without CSH.

Conclusion: Carotid sinus massage at $60^{\circ}$ under controlled conditions should be included in the assessment of patients with non-convulsive faints or unexplained falls. Routine cardiovascular exams, including the HUTT, are not useful for the diagnosis in such cases. Further studies in normal individuals are needed to establish the significance of a positive response to carotid sinus massage in the absence of a history of fainting or falling. (Arq Bras Cardiol 2008; 90(4): 264-267)

Key words: Syncope; hypersensitivity; carotid sinus; tilt table test.

Full texts in English - http://www.arquivosonline.com.br

Correspondência: Marcos Benchimol •

Rua Senador Dantas, 07/601 - Centro - 20031-202 - Rio de Janeiro, RJ - Brasil

E-mail: benchimol@cardiol.br

Artigo recebido em 09/06/07; revisado recebido em 13/09/07; aceito em 30/10/07. 


\section{Introdução}

A descrição original da síncope convulsiva por massagem do seio carotídeo foi também a primeira a interpretar corretamente a bradicardia e a hipotensão como respostas reflexas anormais e sua normalização por aplicação prévia de atropina ${ }^{1}$. Atualmente, a hipersensibilidade do seio carotídeo (HSC) é diagnosticada em pacientes nos quais a massagem do seio carotídeo (MSC) induz assistolia por mais de 3 segundos (tipo cardioinibitório) ou queda da pressão sistólica superior a $50 \mathrm{mmHg}$ (tipo vasodepressor) ${ }^{2}$. Estudos subseqüentes estabeleceram a importância da HSC como causa de desmaios ${ }^{3}$ e ataques de quedas (drop attacks) ${ }^{4}$, assim como o aumento da reatividade reflexa do seio carotídeo com o envelhecimento, principalmente nos homens ${ }^{5}$. A inclusão da MSC no Teste de Mesa Inclinado $(\mathrm{TI})^{6}$ ampliou o leque das causas das síncopes neurocardiogênicas, particularmente as ocasionadas por $\mathrm{HSC}^{7}$. Todavia, a despeito do número crescente de estudos que utilizam a MSC em condições controladas, o significado clínico da HSC permanece obscuro, principalmente em vista de sua prevalência inesperadamente elevada em pessoas que nunca desmaiaram na vida ${ }^{8}$. A presente investigação teve por principais objetivos: 1) reportar uma série de indivíduos encaminhados ao TI para investigação de desmaios ou quedas injustificadas; 2) determinar a prevalência da HSC induzida por MSC nesta série de pacientes, e 3) a utilidade do protocolo do TI utilizado em nosso serviço a partir de um grupo de voluntários sem história de quedas ou desmaios.

\section{Métodos}

Cento e setenta mulheres e 89 homens com idades de 10 a 96 anos (média = $50 \pm 24$ anos) foram encaminhados para investigação de "desmaios" ou quedas inexplicadas ("ataques de quedas") que haviam ocorrido a primeira vez vários meses antes (média $=53 \pm 100$ meses). Dezenove pacientes com MSC + utilizavam regularmente benzodiazepínicos $(n=2)$, anticonvulsivantes $(n=3)$, hipotensores $(n=15)$, diuréticos $(n=5)$ e anticoagulante oral $(n=1)$. O exame físico, ECG e ecocardiograma foram normais ou sem relação com os sintomas de interesse. Cinqüenta e cinco voluntários (32 mulheres e 23 homens) sem história de quedas ou desmaios, com 16 a 88 anos de idade (média $=57 \pm 21$ anos), serviram de controle para o TI. Homens e mulheres não diferiram em idade no grupo de pacientes $(U=5.200, p>0,56)$ ou controles $(U=329, p>0,98)$. Tampouco se constatou diferença entre os grupos quanto à composição de sexo $\left(\chi^{2}=\right.$ $0,77, p>0,38)$. Todos forneceram consentimento informado por escrito antes do ingresso no estudo, que foi aprovado pelo Comitê de Ética do Hospital Universitário Gaffrée e Guinle.

\section{Teste inclinado e MSC}

O TI foi realizado entre 14 e 17 horas $^{9}$, em sala silenciosa, iluminação tênue e temperatura ambiente agradável, de acordo com recomendações técnicas amplamente aceitas ${ }^{10}$. A mesa de exame (Carci, São Paulo, Brasil), equipada com sistema TEB para monitoração contínua do ECG e apoio para os pés, permite inclinações de 60 a $80^{\circ}$. A pressão arterial foi aferida por aparelho oscilométrico e os valores confirmados com esfigmomanômetro a intervalos regulares.
Bandeja de reanimação foi mantida ao alcance por todo o tempo de exame. Solicitou-se a cada indivíduo que cumprisse jejum de 12 horas e, quando possível, que suspendesse os medicamentos 24 horas antes. Após 10 minutos de repouso em decúbito dorsal, o examinador massageava manualmente por 5 segundos a região do pescoço onde a pulsação da carótida atingia intensidade máxima, geralmente no plano da borda superior da cartilagem cricóide, começando pelo lado direito. Em seguida, inclinava a mesa a $60^{\circ} \mathrm{e}$ repetia a manobra de cada lado. Observações anteriores mostraram que a massagem dos seios carotídeos pode ser inteiramente normal a zero grau e tornar-se positiva apenas após inclinação ${ }^{11}$. O TI consistiu de duas condições ${ }^{10}:$ 1) uma condição "passiva" (isto é, sem intervenção farmacológica) e 2) uma condição farmacologicamente "sensibilizada", em que se ministrava 1,25 mg de dinitratro de isosorbida por via sublingual, para provocar sintomas inaparentes na condição passiva. Cada condição durava 25 minutos ou menos, em caso de pré-síncope. Quando positivo na primeira fase, concluía-se o teste sem passar à condição sensibilizada. O TI foi conduzido normalmente nos casos de MSC positiva. O TI foi considerado anormal ("positivo") se sintomas sincopais ou pré-sincopais mediados por mecanismos neurais ocorressem por causa de hipotensão, bradicardia, ou ambos, quer o indivíduo atestasse ou não a replicação dos sintomas que o acometiam na vida real. A MSC foi considerada positiva se houvesse queda mínima de $50 \mathrm{mmHg}$ da pressão arterial sistólica ou bradicardia/asistolia ${ }^{2,10}$. Sopro carotídeo, acidentes cerebrovasculares ou infarto agudo do miocárdio nos seis meses precedentes ou história de arritmia ventricular grave constituíram contra-indicação para a realização do exame.

\section{Análise estatística dos resultados}

Os resultados são descritos por médias e desvios padrão. Possíveis associações entre variáveis categóricas foram inferidas pelo teste do $\chi^{2}$. A significância de diferenças entre grupos foi aferida pelos testes de Mann-Whitney $(U)$ e Exato de Fisher ${ }^{12}$. A contribuição relativa de idade, sexo, resultado do TI, e índices fisiológicos (pressão arterial e freqüência cardíaca) para ocorrência da HSC foi inferida por regressão logística binomial. A regressão logística binomial é apropriada para variáveis dependentes dicotômicas (por exemplo, MSC+ vs. MSC-), às quais se atribui valor 1 à de principal interesse (por exemplo, MSC+) e zero à outra (por exemplo, MSC-). O tamanho do efeito e o sentido da associação entre as variáveis independentes são expressos como odds ratios (OR). Cada or pode variar de zero a 0,999 (no caso de uma relação inversa) ou de 1,001 ao infinito (no caso de uma relação direta); quando $\mathbf{O R}=1$, as variáveis são estatisticamente independentes ${ }^{13}$. O tamanho do efeito global da regressão logística foi aferido pelo coeficiente $\mathrm{R}^{2}$ de Nagelkerke, que, à semelhança do coeficiente de determinação múltipla $\left(R^{2}\right)$, varia entre zero e 1. Adotamos limiar de significância de 0,05, bicaudado, para todos os testes estatísticos. Os cálculos foram realizados no spss for Windows, v. 13.0 (sPss Inc, 2004).

\section{Resultados}

Os principais resultados estão apresentados na Tabela 1. Dos 


\section{Artigo Original}

Tabela 1 - Características demográficas e afiliações clínicas e hemodinâmicas à estimulação dos seios carotídeos

\begin{tabular}{|c|c|c|}
\hline & $\begin{array}{l}\text { Manobra vagal positiva } \\
\text { (HSC) } N=28\end{array}$ & $\begin{array}{c}\text { Manobra vagal negativa } \\
\qquad \mathrm{N}=231\end{array}$ \\
\hline Idade $(\text { anos) })^{*}$ & $71 \pm 11$ & $47 \pm 23$ \\
\hline $\operatorname{Sexo}(M / F)^{*}$ & $20 / 8$ & $69 / 162$ \\
\hline $\begin{array}{l}\text { Freqüência de } \\
\text { desmaios }\end{array}$ & $4,4 \pm 3,4$ & $3,7 \pm 2,7$ \\
\hline $\begin{array}{l}\text { Duração dos } \\
\text { sintomas (meses) }\end{array}$ & $59 \pm 88$ & $52 \pm 94$ \\
\hline $\begin{array}{l}\text { Teste Inclinado } \\
\text { (positivo/normal) }\end{array}$ & $20 / 8$ & $149 / 82$ \\
\hline Passivo (min) & $22 \pm 8$ & $24 \pm 4$ \\
\hline Sensibilizado (min) & $13 \pm 10$ & $15 \pm 9$ \\
\hline \multicolumn{3}{|l|}{ FC } \\
\hline basal & $67 \pm 8$ & $72 \pm 12$ \\
\hline máxima & $88 \pm 15$ & $101 \pm 18$ \\
\hline durante $^{* *}$ & $50 \pm 25$ & $65 \pm 24$ \\
\hline \multicolumn{3}{|l|}{ PA sistólica } \\
\hline basal & $131 \pm 28$ & $121 \pm 21$ \\
\hline máxima & $137 \pm 28$ & $123 \pm 26$ \\
\hline durante & $65 \pm 21$ & $70 \pm 17$ \\
\hline \multicolumn{3}{|l|}{ PA diastólica } \\
\hline basal & $80 \pm 14$ & $74 \pm 13$ \\
\hline máxima & $84 \pm 14$ & $80 \pm 15$ \\
\hline durante & $34 \pm 13$ & $40 \pm 13$ \\
\hline
\end{tabular}

259 pacientes encaminhados ao TI, 28 (11\%) apresentaram MSC + a $60^{\circ}$ e a zero grau apenas em três. Nenhum participante apresentou complicações da MSC e do TI, o que vai ao encontro da segurança reportada do teste ${ }^{14}$.

\section{Pacientes}

Quando comparados aos pacientes MSC-, o grupo de pacientes MSC+ se constituiu predominantemente de homens idosos. Embora o TI tenha sido positivo em mais de dois terços (71\%) destes últimos, essa proporção não diferiu estatisticamente da observada nos pacientes MSC- $(p=0,53)$.

Para explorar as associações clínicas da HSC, procedemos a regressões logísticas binomiais computando a MSC (MSC+ vs. MSC-) como variável dependente e, como variáveis independentes, idade, sexo, história de desmaios (pacientes vs. controles), resultado do TI (positivo vs. normal) e índices hemodinâmicos (pressão arterial e freqüência cardíaca). Houve correlação significativa entre o conjunto de variáveis e a ocorrência de HSC $\left(\mathrm{R}^{2}=0,47, p<0,001\right)$. A HSC foi prevista por sexo masculino (OR $=5,19, p<0,02$ ), idade avançada ( $\mathbf{O R}=1,10, p<0,001)$ e menor freqüência cardíaca durante o $\mathrm{TI}$ no grupo com MSC+ $(\mathrm{OR}=0,96, p<0,04)$.
Esses resultados não se modificaram qualitativamente após remoção dos controles da planilha de cálculo.

Em análise subseqüente, verificamos o resultado da MSC apenas nos pacientes com mais de 60 anos de idade ( $n=138$ ). Nesse subgrupo, a MSC foi positiva em 27, ou 19\% (20 homens), e negativa em 111, ou 81\% (32 homens). Como antecipado, não houve diferença estatística de idade entre os grupos $(U=1411, p>0,64)$, mas a predominância masculina de HSC foi reafirmada $\left(\chi^{2}=19, p<0,001\right)$.

\section{Controles}

No grupo controle, a MSC a $60^{\circ}$ foi positiva em quatro homens com idade mediana de 70 anos. O predomínio masculino foi significativo nesse grupo em relação aos demais controles ( $p=0,02)$, e, embora as idades não diferissem ( $U$ $=73, p>0,45)$, esse grupo também mostrou tendência a menor freqüência cardíaca basal durante o TI (medianas: 62 bpm x $70 \mathrm{bpm}(U=41,5, p<0,07))$. O TI foi positivo em apenas três controles $(<6 \%)$, nenhum dos quais apresentou HSC. Em relação aos controles, a positividade do TI foi significativamente maior nos pacientes com $\operatorname{HSC}\left(\chi^{2}=35\right.$, $p<0,001)$.

\section{Discussão}

Os principais resultados do estudo podem ser assim resumidos: a) dentre os pacientes com desmaios, o TI não diferiu naqueles com e sem HSC; b) estatisticamente, a freqüência de HSC não diferiu entre pacientes e controles; c) a propensão à HSC nos homens idosos relacionou-se, estatisticamente, à idade avançada e ao sexo masculino; d) a aplicação da MSC a zero grau pode ser dispensada em favor da aplicação da MSC a $60^{\circ}$.

\section{Importância da MSC no diagnóstico da HSC}

Nossos achados enfatizam a importância da realização da MSC a $60^{\circ}$ no diagnóstico da HSC: em 259 pacientes com história de desmaios ou quedas inexplicadas, 28 (11\%) apresentaram HSC à inclinação de $60^{\circ}$. Destes, em apenas três a resposta foi positiva a $0^{\circ}$. Em contraste, apenas quatro controles apresentaram HSC. Curiosamente, a HSC não foi maior no grupo com história de desmaios em relação aos controles. Embora os quatro indivíduos do grupo controle com HSC pudessem vir a apresentar desmaios se acompanhados por tempo suficiente, por ora podemos apenas sugerir que a utilidade diagnóstica da MSC + deve se restringir àqueles indivíduos com história de desmaios claramente relacionados a manipulações cervicais. A relevância da HSC é maior quando coincide com história de desmaios ou quedas precipitadas por manipulações cervicais na vida real. Infelizmente, o nexo causal entre posição da cabeça e síncope fica nublado pela amnésia transitória que acomete alguns desses pacientes ${ }^{15}$. Estudo recente em 80 indivíduos assintomáticos com mais de 65 anos constatou HSC em 28 (35\%) deles ${ }^{16}$. A discrepância entre este resultado e o do presente estudo provavelmente se deve à idade média mais baixa e à maior prevalência de mulheres nos nossos controles. Embora nossos resultados e os de vários outros estudos concordem que a HSC é 
ocorrência relativamente freqüente em indivíduos que nunca desmaiaram, o significado exato desse fenômeno apenas será esclarecido por estudos prospectivos que determinem a incidência de desmaios com a passagem do tempo. Por enquanto, a MSC+ na ausência de história de desmaio ou queda detém significado clínico incerto ${ }^{2,15}$.

\section{Teste inclinado e MSC}

A positividade do TI não diferiu entre pacientes com e sem HSC, isto é, o TI não substituiu a MSC a $60^{\circ}$ no diagnóstico da HSC. Do ponto de vista prático, se a MSC não fosse realizada nos 8 indivíduos com desmaios e TI negativo, o diagnóstico de HSC passaria despercebido. Além disso, os pacientes com HSC e TI negativo indicam a operação de mecanismos fisiopatológicos distintos na produção da síncope.

De fato, os mecanismos fisiopatológicos exatos que levam à falha intermitente dos reflexos cardiovasculares no dia-adia dos pacientes com HSC ainda não foram completamente esclarecidos. Nesse sentido, uma constatação fisiopatológica potencialmente relevante foi a menor FC durante o $\mathrm{TI}$ nos pacientes com HSC, sugestiva de falha nos reflexos baroceptores. Esses reflexos, cuja fisiologia é hoje bem conhecida ${ }^{2}$, são integrados por fibras que chegam pelos nervos glossofaríngeos aos núcleos vegetativos da formação reticular do bulbo, de onde emanam fibras vagais e retículo-espinhais para a coluna simpática da medula torácica ${ }^{17}$. Novos estudos são necessários para esclarecer os segmentos alterados desse circuito que comprometem a resposta cronotrópica nos pacientes com HSC.

\section{Contribuição da MSC para a economia diagnóstica}

A maior parte dos pacientes com HSC havia consultado generalistas e especialistas antes que o TI fosse solicitado. É provável que a imprevisibilidade e a raridade dos desmaios tenham contribuído para retardar o diagnóstico de HSC. A média de quatro desmaios, aparentemente pequena em número absoluto, não deve subestimar a elevada morbidade associada a uma única queda, principalmente em pessoas idosas ${ }^{18}$. Além disso, o atraso no diagnóstico implica custos freqüentemente desnecessários. Por exemplo, se os pacientes encaminhados a parecer de especialista (cardiologista ou neurologista), eletrocardiograma e ecocardiograma, Holter, EEG, ecodoppler de carótidas e vertebrais, e pelo menos um exame de imagem cerebral (tomografia computadorizada ou ressonância magnética) houvessem sido submetidos no início à MSC, o custo diagnóstico seria substancialmente menor. Aparentemente, tal não ocorreu por falta de uma estratégia diagnóstica guiada pela hipótese de HSC nesses casos.

Ainda do ponto de vista econômico, a HSC encerra implicações adicionais para a condução de veículos automotores ${ }^{19}$, pois, em razão do mecanismo de deflagração dos desmaios nesses casos, essa modalidade de síncope pode acometer o indivíduo sentado ao volante, em movimentos da cabeça que comprimam um dos seios carotídeos anormalmente sensível.

\section{Agradecimentos}

Os autores agradecem ao Professor Omar da Rosa Santos (Hospital Universitário Gaffré e Guinle, Rio de Janeiro) pelo apoio institucional e ao Srs. José Ricardo Pinheiro e Jorge Baçal (Instituto Oswaldo Cruz, Rio de Janeiro) pela sua inestimável ajuda na pesquisa da literatura pertinente. Esse estudo deve muito à inspiração intelectual do Sr. Emilio Sunda, atualmente no Physiological Department of the University of Manchester, UK.

\section{Potencial Conflito de Interesses}

Declaro não haver conflito de interesses pertinentes.

\section{Fontes de Financiamento}

O presente estudo não teve fontes de financiamento externas.

\section{Vinculação Acadêmica}

Não há vinculação acadêmica deste estudo a programas de pós-graduação.

\section{Referências}

1. Roskam J. Syncopes cardiaques graves et syncopes répétées par hyperréflectivité sino-carotidienne. Press Méd. 1930; 38: 590-1.

2. Brignole M, Alboni P, Benditt DG, Bergfeldt L, Blanc JJ, Thomsen PE, et al Guidelines on management (diagnosis and treatment) of syncope-update 2004: executive summary. Eur Heart J. 2004; 25: 2054-72.

3. Nahm F, Freeman R. Syncope and the history of nervous influences on the heart. Arch Neurol. 2003; 60: 282-7.

4. Parry SW, Kenny RA. Drop attacks in older adults: systematic assessment has a high diagnostic yield. J Am Ger Soc. 2005; 53: 74-8.

5. Smiddy J, Lewis HD, Dunn M. The effect of carotid massage in older men. J Gerontol. 1972; 27: 209-11.

6. Kenny RA, Ingram A, Bayliss J, Sutton R. Head-up tilt: a useful test for investigating unexplained syncope. Lancet. 1986; 1: 1352-5.
7. Grubb BP, Kosinski D. Tilt table testing: concepts and limitations. Pacing Clin Electrophysiol. 1997; 20: 781-7.

8. McGlinchey PG, Armstrong L, Spence MS, Roberts MJ, McKeown PP. Effect of carotid sinus massage and tilt-table testing in a normal, healthy older population (The Healthy Aging Study). Am J Cardiol. 2002; 90: 1015-7.

9. Wu TC, Hachul D, Scanavacca M, Sosa E. Comparison between tilt-table testing results performed during different periods of the day. Arq Bras Cardiol. 2002; 79: 390-4.

10. Benditt DG, Ferguson DW, Grubb BP, Kapoor WN, Kugler J, Lerman BB, et al. Tilt table testing for assessing syncope. J Am Coll Cardiol. 1996; 28 (1): 263-75.

11. Benchimol M, Oliveira-Souza R. Syncope in the elderly: diagnostic utility of carotid sinus massage in the head-up tilt test. Arq Neuropsiquiatr. 2003; 61: 87-90. 


\section{Artigo Original}

12. Siegel S, Castellan NJ. Nonparametric statistics for the behavioral sciences, 2nd ed. New York: McGraw-Hill, 1988.

13. Sandercock P. The odds ratio: a useful tool in neurosciences. J Neurol Neurosurg Psychiatry. 1989; 52 (7): 817-20.

14. Coplan NL. Carotid sinus hypersensitivity and syncope: Cause/effect or true/true/unrelated. Arch Intern Med. 2006; 166: 491-2.

15. Cummings SR, Nevitt MC, Kidd S. Forgetting falls: the limited accuracy of recall of falls in the elderly. J Am Geriatr Soc. 1988; 36: 613-6.
16. Kerr SRJ, Pearce MS, Brayne C, Davis RJ, Kenny RA. Carotid sinus hypersensitivity in asymptomatic older persons: implications for diagnosis of syncope and falls. Arch Intern Med. 2006; 166: 515-20.

17. Craig AD. Forebrain emotional asymmetry: a neuroanatomical basis? Trends Cogn Sci. 2005; 9: 566-71.

18. Tinetti ME. Preventing falls in elderly persons. N Engl J Med. 2003; 348: 42-9.

19. Li H, Weitzel M, Easley A, Barrington W, Windle J. Potential risk of vasovagal syncope for motor vehicle driving. Am J Cardiol. 2000; 85: 184-6. 